Check for updates

Cite this: RSC Adv., 2017, 7, 38812

\title{
Defining the role of nanonetting in the electrical behaviour of composite nanofiber/nets $\uparrow$
}

\author{
G. Massaglia, ${ }^{\star a b}$ A. Chiodoni, (DD a G. P. Salvador, ${ }^{a}$ L. Delmondo, ${ }^{b}$ J. A. Muñoz-Tabares, ${ }^{a}$ \\ S. Bocchini, (iD ${ }^{\text {a }}$ A. Sacco, (D) ${ }^{\text {a }}$ S. Bianco, ${ }^{\text {ab }}$ G. Saracco ${ }^{a}$ and M. Quaglio (DD *a
}

This work investigates the effect of nanofiber/nets (NFN) on the electrical behaviour of conductive composite nanofibers, using polyethylene oxide (PEO)/multiwall carbon nanotubes (MWCNT) as the model material for the analysis. We demonstrate that water-based solutions, containing high molecular weight PEO and MWCNT can be processed by electrospinning/netting applying high voltages. NFNs are designed with different morphologies, in particular modulating their porosities. A strong enhancement of the electrical conductivity of PEO/MWCNT-NFN is observed for the denser NFN, if compared to nanofiber composites. These results demonstrate that the NFN morphology can directly control the quality of the electron percolation paths. To provide better evidence for this strong dependency we processed the nanocomposite NFN by solvent-induced swelling. This physical mechanism directly modulates the percolation path, tuning the electrical behaviour of the nanocomposites to a resistive one: the better the starting percolation path, the larger the resistivity increase.

Received 17th May 2017

Accepted 2nd August 2017

DOI: $10.1039 / \mathrm{c} 7 \mathrm{ra05573k}$

rsc.li/rsc-advances calcium carbonate, ${ }^{\mathbf{8 , 1 0 , 1 1}}$ cellulose ${ }^{5}$ and carbon nanotubes, ${ }^{12-14}$ have been successfully used to induce the ESN process. Except for salts, the other additives act as fillers in the final nanostructure, thus forming NFN-composites with significantly improved mechanical properties. Interestingly, even if electrically conducting fillers (i.e., carbon nanotubes) have been used as additives to promote the ESN process, a substantial lack of information exists on the electrical behaviour of the resulting NFN nanocomposite.

In this paper we describe the nanocomposite obtained by adding multiwall carbon nanotubes (MWCNTs) to a waterbased solution of polyethylene oxide (PEO). Sodium polystyrene sulfonate (Na-PSS) was chosen as surfactant agent, as reported in former works on MWCNTs, ${ }^{15-17}$ in order to effectively dispersed MWCNT in the initial solution and in the following products. The resulting fibrous nanocomposite was the model material to investigate the behaviour of electrically conductive NFN-nanocomposites. The electrical properties of PEO/MWCNT nanofibers have been deeply investigated by McCullen et al. ${ }^{18}$ demonstrating that the MWCNT concentration directly modulates the electrical conductivity, according to the percolation model. In this kind of composite material, the actual morphology of the polymer nanofibers hosting the conductive filler can strongly influence the creation of efficient percolation paths; therefore the presence of NFN can deeply modify its electrical behaviour.

We firstly demonstrate the possibility to obtain NFN by electrospinning/netting of PEO/MWCNT nanocomposite, proving that they can form when using high molecular weight PEO and high voltages. By controlling the process parameters, 
we obtained different morphologies of the NFN, especially in terms of density and shape of nanonets.

We then analysed the electrical behaviour of PEO/MWCNTNFN, confirming the validity of the percolation model in this system. We demonstrated that the morphology of the NFN system deeply influences its electrical behaviour, showing also the possibility to modulate the percolation path in NFN nanocomposites, by exploiting the effect of solvent (water)-induced swelling. Indeed, by swelling PEO-based nanofibers and nanonets with water, we changed their average diameters, thereby increasing the average distance among MWCNTs. This physical mechanism directly enhances the resistive behaviour of the nanocomposite: the better the starting percolation path, the larger the resistivity increase.

\section{Experimental}

\section{Materials}

Two electrospinning solutions at $5 \mathrm{wt} \%$ in deionized (DI) water were prepared using PEOs with different average molecular weight $600 \mathrm{kDa}$ and $1000 \mathrm{kDa}$ (from Sigma Aldrich). The corresponding solutions are named PEO-600 and PEO-1000, respectively. Each solution was aged overnight under continuous stirring at room temperature. Conductive composite nanofibers were obtained by adding different mass fraction of MWCNTs to the prepared PEO solutions. The complete process flow is represented in Fig. 1. MWCNTs (NC3100 by Nanocyl) with purity higher than $95 \%$ and average length of $1.5 \mu \mathrm{m}$, were dispersed in DI water and sodium polystyrene sulfonate (Na-PSS) by Sigma Aldrich, to ensure a better dispersion (Na-PSS to MWCNTs molar ratio equal to 1$)$ by ultrasonic bath (2000U Digital Sonifier by Branson). In Fig. 1(a) a sketch is proposed, describing the preparation of PSS functionalized MWCNTs. Solutions with different weight percentage of functionalized MWCNTs, from 1 to $7 \mathrm{wt} \%$, were prepared. Water-based solutions of PEO where mixed with water-based MWCNT/PSS solutions, as described in Fig. 1(b), and aged under continuous stirring overnight at room temperature, to obtain the final input

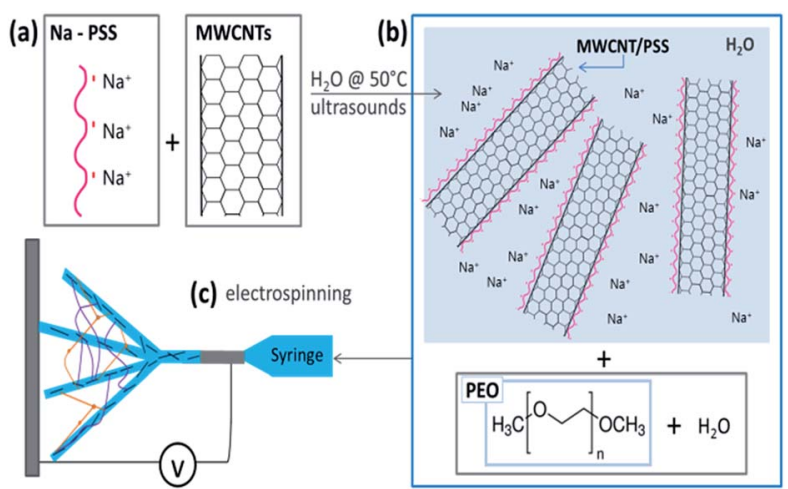

Fig. 1 Process flow for the preparation of MWCNT/PSS/PEO nanofibers and nanofiber/nets. (a) MWCNTs are functionalized by PSS in water, at $50^{\circ} \mathrm{C}$ in ultrasonic bath. (b) The resulting solutions are mixed to water-based PEO solutions. (c) Final samples are fabricated by electrospinning. solution for the electrospinning step, as shown in Fig. 1(c). The viscosity was evaluated by rheometric analysis (MCR Rheometer series Anton Paar).

\section{Electrospinning}

The electrospinning equipment NANON 01A by MECC was used. It allows the flow rate tuning from $0.1 \mathrm{ml} \mathrm{h}^{-1}$ to $99.9 \mathrm{ml} \mathrm{h}^{-1}$, and the operating voltage, from $0.5 \mathrm{kV}$ to $30 \mathrm{kV}$. The solutions were loaded in a syringe of $6 \mathrm{ml}$, connected to a needle $(27$ gauge $\times 15 \mathrm{~mm})$.

Aluminum foils were used as the reference substrates during process optimization. Samples for TEM analysis were prepared by directly electrospinning the nanofibers on a standard holey carbon Cu TEM grid.

\section{Nanofibers characterization}

The morphology of the nonwoven mats was deeply characterized by Field Effect Scanning Electron Microscopy (FESEM, Zeiss Merlin). To investigate the dispersion of MWCNTs in the composite nanofibers mat. Raman Spectroscopy (Renishaw 1000 spectrometer) and Transmission Electron Microscopy (TEM) were performed. In this work, a FEI Tecnai F20ST equipped with a field emission gun, operating at $200 \mathrm{kV}$, was used. The thermal properties of the composites NN and NFN were measured by Thermo-Gravimetric-Analysis (Netzsch TG 209 F1) under inert atmosphere.

Glass slides were used as substrates to fabricate the samples for electrical characterizations. Hard-masks, made of poly (methyl)methacrylate were fabricated by column drill, and used both to prepare the electrodes and to shape the nanofibers mats for the characterizations. Strips of nanofibers were prepared, having a length of $2 \mathrm{~cm}$ and a width of $2 \mathrm{~mm}$. The thickness of the nanofiber strips was evaluated by a Tencor P-10 Surface Profiler and confirmed by FESEM analysis on samples analysed in cross-section. Four platinum electrodes were obtained for each nanofiber strip, using a hard mask during sputtering from a Pt target, applying a current of $50 \mathrm{~mA}$ for $120 \mathrm{~s}$ (Q150T ES by Quorum Technologies). The resulting platinum films were $100 \mathrm{~nm}$ thick, $1 \mathrm{~mm}$ wide and $5 \mathrm{~mm}$ long. The four-points electrical characterizations were performed by polarising the samples, applying different values of electrical potential difference $(V)$, and measuring the resulting current $(I)$ flowing through the sample by means of a Keithley 2635A Source measure unit.

\section{Results and discussion}

Works discussing the electrospinning of water-based solutions containing PEO and carbon nanotubes are available in the literature, ${ }^{\mathbf{1 8 - 2 0}}$ none of them reporting the formation of 2D-nanonets from those solutions, even if additives (including PSS) were added to favour the MWCNT dispersion.

In the present work, we promoted the electrospinning/ netting process by adding high molecular weight $\left(M_{\mathrm{w}}\right)$ PEO to a water-based solution containing PSS-functionalized MWCNTs, and by applying high voltages during the electrospinning process. 
The detailed composition of all the solutions tested in this work, as well as their electrical conductivities and rheological behaviour, are listed in Table S-1 in the ESI. $\dagger$ We obtained ESN process using PEO with the highest molecular weight (i.e., $1000 \mathrm{kDa}$ ) at the highest concentration (i.e., $5 \mathrm{wt} \%$ ). No nanonets were observed for lower working parameters, as reported in the Fig. S3† in the ESM. The name of the nanocomposite samples has the general structure PEO- $x-y @ z$, where $x$ is the polymer $M_{\mathrm{w}}, y$ refers to the amount of MWCNTs and $z$ refers to the voltage applied during the electrospinning process. When a class of samples with different concentrations of MWCNTs is considered, a specific value will not be assigned to $y$.

We hypothesized that the ESN is promoted by the combined effect of Na-PSS additive and high molecular weight PEO. In Fig. 1 the PSS is simplified by a pink line with negative charges: when Na-PSS is added to MWCNTs in water, PSS links to the MWCNT surface, exposing the negative charges, well balanced by the $\mathrm{Na}^{+}$ions, to water, as proposed in Fig. 1(a). By adding PEO to the solution, $\mathrm{Na}^{+}$are attracted by the ether groups. The resulting mobility of positive and negative charges into the final polymeric solution is reduced. When the electric field is applied, the polymeric solution at the tip of the needle can expose unbalanced local concentration of the positive $\mathrm{Na}^{+}$ions linked to the PEO ether groups, and the negatively charged PSS-MWCNTs, so increasing the probability of charge unbalances inside the nanofibers, as sketched in Fig. 2(a). Interestingly, when PEO with a molecular weight of $600 \mathrm{kDa}$ is used, no NFNs form, even if using high voltages (see Fig. S-3a $\dagger$ in the ESM). When the molecular weight is increased to $1000 \mathrm{kDa}$, NFNs form, with a structure that becomes more complex for increasing values of the applied voltage and for higher concentration of Na-PSS/MWCNTs, as shown in Fig. 2(b). Therefore, we suggest that the use of high $M_{\mathrm{w}}$ is critical to favour random, unbalanced distribution of charges, and then to form NFN, as proposed in Fig. 2(a). Indeed, it is important to consider that, thanks to the high number of entanglements present in the high $M_{\mathrm{w}}$ PEO, it is quite easy to create charge unbalances by the reduced ion mobility during the fast ESN process.

Different net charge distributions between adjacent nanofibers can significantly contribute to the creation of strong local secondary electric fields that can generate the secondary nanofiber net, as sketched in Fig. 2a. The result is the formation of nanofiber/nets, according to a mechanism similar to that proposed by N. A. M. Barakat et al., ${ }^{9}$ based on the effect of salt additives.

In order to analyse the actual distribution and the orientation of MWCNTs inside the nanofibers-net, and to gain a better understanding of the morphology of the PEO/MWCNT-NFN, TEM analyses were performed. We selected $23 \mathrm{kV}$ and $0.1 \mathrm{ml} \mathrm{h}^{-1}$ as the reference electrospinning parameters and fixed the MWCNT loading at $1.5 \mathrm{wt} \%$. Nanofiber/nets were directly collected on standard holey carbon TEM Cu grid. Fig. 2(c) and (d) help to confirm that the MWCNTs are aligned along the main axis of the nanofibers, showing the presence of MWCNTs even in small-size nanofibers.

The morphology of the NFNs obtained for the nanocomposite samples are shown in Fig. 3. FESEM pictures of samples PEO-1000-1.5@18 and PEO-1000-1.5@23 are shown in Fig. 3(a) and (b) respectively, while samples PEO-1000-3.5@18 and PEO-1000-3.5@23 are shown in Fig. 3(c) and (d), respectively. The NFNs are highlighted in yellow, showing the creation of a secondary web, made of very small-size nanofibers. By increasing the voltage up to $23 \mathrm{kV}$ and the MWCNT concentration up to $3.5 \mathrm{wt} \%$, the nanofiber-net morphology becomes more complex. Indeed, the comparison of the first line with respect to the second line of Fig. 3, makes it clear that the
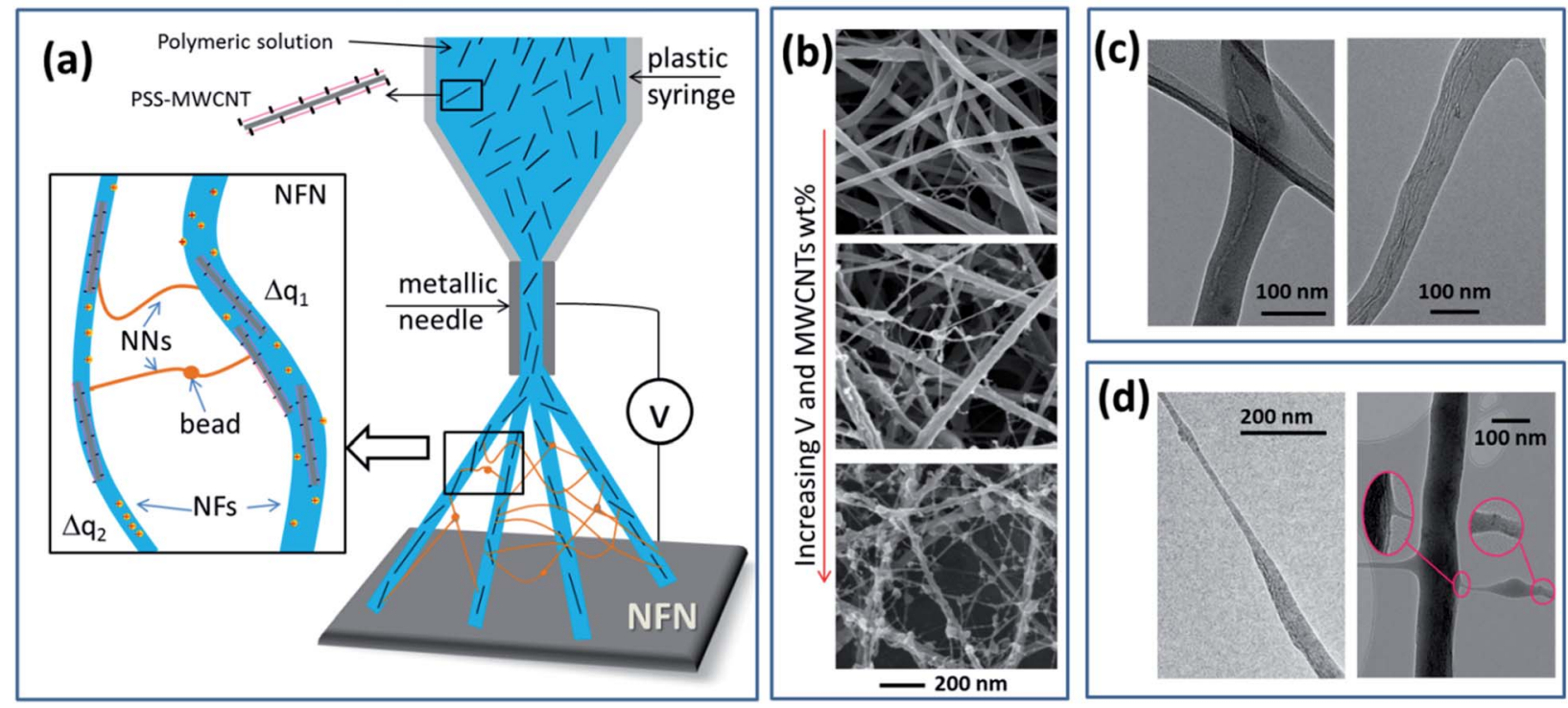

Fig. 2 In (a) the electrospinning/netting process is sketched, highlighting the effect of charge unbalances in the creation of nanobeaded NFN structure. In (b) FESEM images of NFNs are shown, evidencing the effect of increasing voltage and MWCNT concentration. In (c) tem analysis of nanocomposite nanofibers with $1.5 \mathrm{wt} \%$ MWCNTs is proposed, showing MWCNTs well aligned with the main axis of the nanofibers. In (d) a TEM images of small size nanofibers and nanobeaded-NFN are shown. 

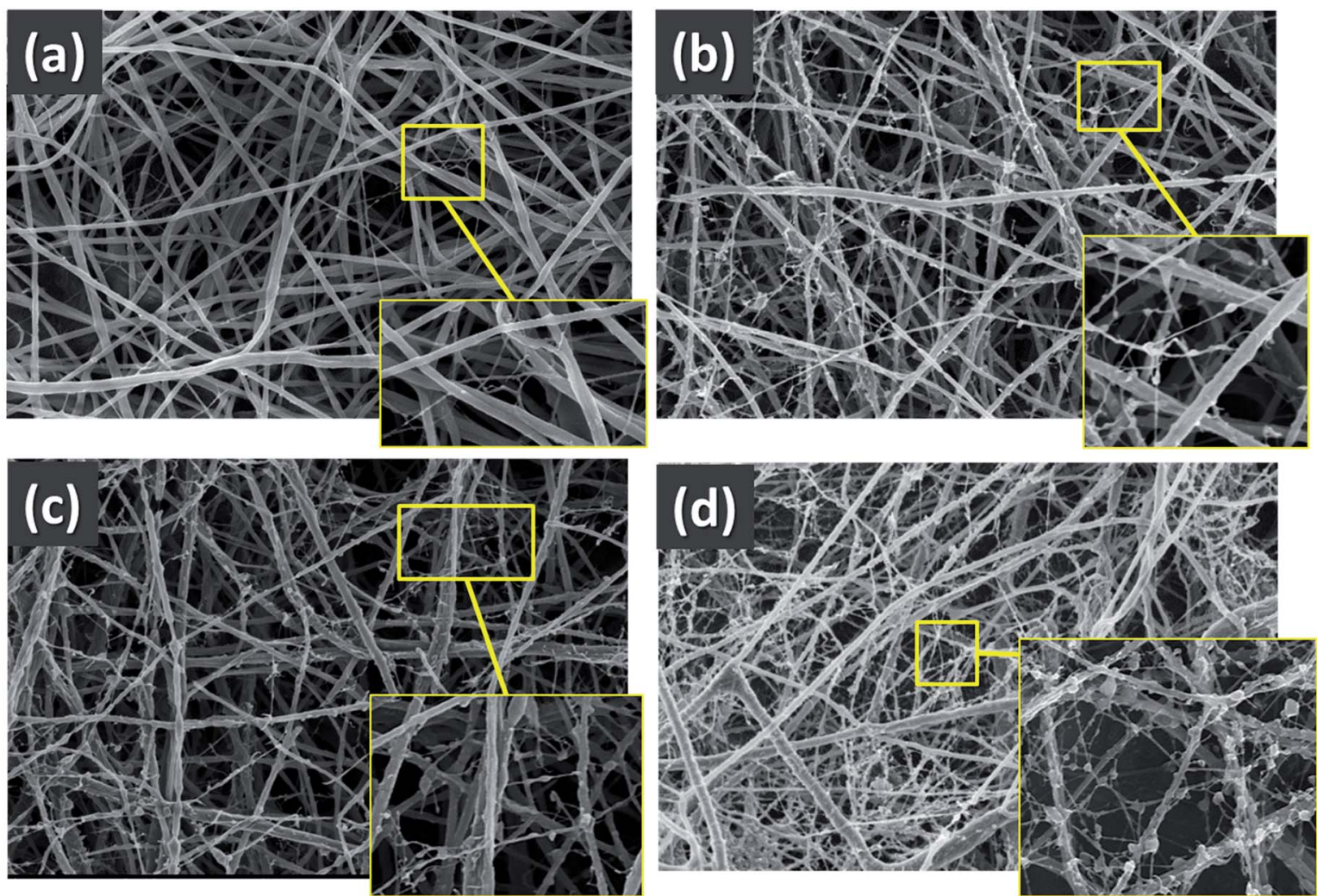

main pictures

$1 \mathrm{~mm}$

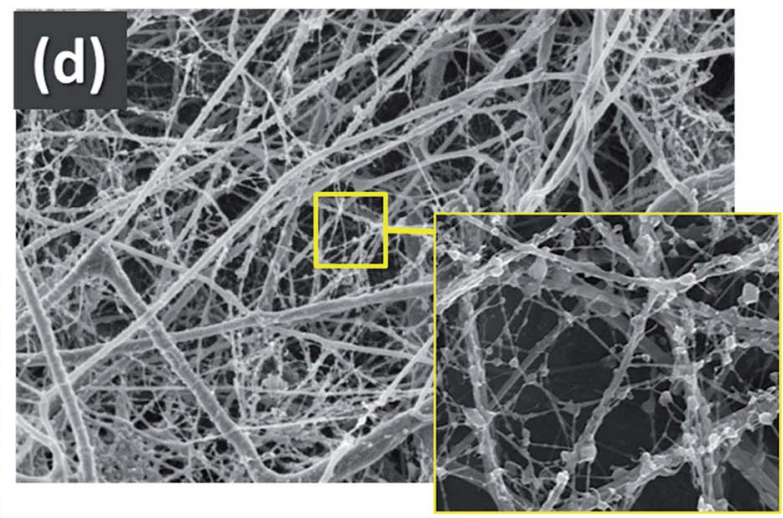

details $-200 \mathrm{~nm}$

Fig. 3 Lines report FESEM images describing the effect of MWCNT concentration on the NFN morphology, while columns describe the effect of voltage. In more details: (a) and (b) show the NFNs obtained using 1.5 wt\% MWCNTs, and (c) and (d) show the NFN referring to 3.5 wt\% MWCNTs. In (a) and (c) the NFNs obtained at $18 \mathrm{kV}$ are proposed, while the effect of $23 \mathrm{kV}$ is proposed in (b) and (d).

nanofiber nets are denser in the composite systems having a higher content of MWCNTs, meaning that more nanonets form connecting the main nanofibers, occupying the inner pores of the material. This observation is consistent with the hypothesis that MWCNTs rule the NFN creation process. Comparing the first column of Fig. 3 to the second one, the effect of increasing values of the working voltage is appreciable, especially showing the formation of nanobeads, distributed along the nanonets, as shown in Fig. 3. We will refer to this structure as nanobeaded-NN. The concentration of nanobeaded-NN increases both with the concentration of MWCNTs and the applied voltage.

We consider MWCNTs to play a key role in determining the nanobeaded morphology of the PEO/MWCNT-NFN. As already investigated in electrospinning, ${ }^{21}$ beads can form during the travel of the fluid jet, if the driving force elongating the nanofiber is reduced. Thus, we suggest that unbalanced charges, due to MWCNTs, alter the actual electrical field applied to the nanofibers, so favouring the creation of nanobeaded-NN as evidenced by the TEM analysis reported in Fig. 2(d).

A percolation behaviour has been demonstrated by McCullen et al. for the PEO/MWCNT nanofiber composite. ${ }^{18}$ To investigate the role of nanonets on the electrical behaviour of the PEO/MWCNT-NFN nanocomposite, some samples were fabricated using solutions made of both a low and a high PEO molecular weights (i.e., PEO-600 and PEO-1000 samples, respectively), with different mass fractions of MWCNTs. Since no NFN has been observed in PEO-600 samples (as proposed in the ESI $\dagger$ ), we selected them as the reference to show the electrical behaviour of standard NF, comparing them with the NFN samples obtained using the 1000 KDa PEO. Moreover, PEO-600 samples were processed at $18 \mathrm{kV}$ only, while the PEO-1000 samples were fabricated at two different voltages, i.e. 18 and $23 \mathrm{kV}$, in order to obtain samples with different nanonets densities, as reported in Fig. 3.

The experimental values of the electrical conductivity as a function of the MWCNT weight percentages are represented in Fig. 4(a). We fitted the three sets of data with the percolation models reported in eqn (1) and (2), that describe the electrical conductivity as a function of the filler content above and below the percolation threshold, respectively.

$$
\begin{aligned}
& \sigma=\sigma_{0}\left(p-p_{\mathrm{c}}\right)^{s} \\
& \sigma=\sigma_{\mathrm{d}}\left(p_{\mathrm{c}}-p\right)^{t}
\end{aligned}
$$

In the model, $p_{\mathrm{c}}$ is the percolation threshold, $\sigma$ is the electrical conductivity, $p$ is the weight percentage of MWCNTs, and $\sigma_{0}, \sigma_{\mathrm{d}}, t$ and $s$ are fit parameters. The fitting curves are proposed in Fig. 4(a), and the resulting values of the percolation 

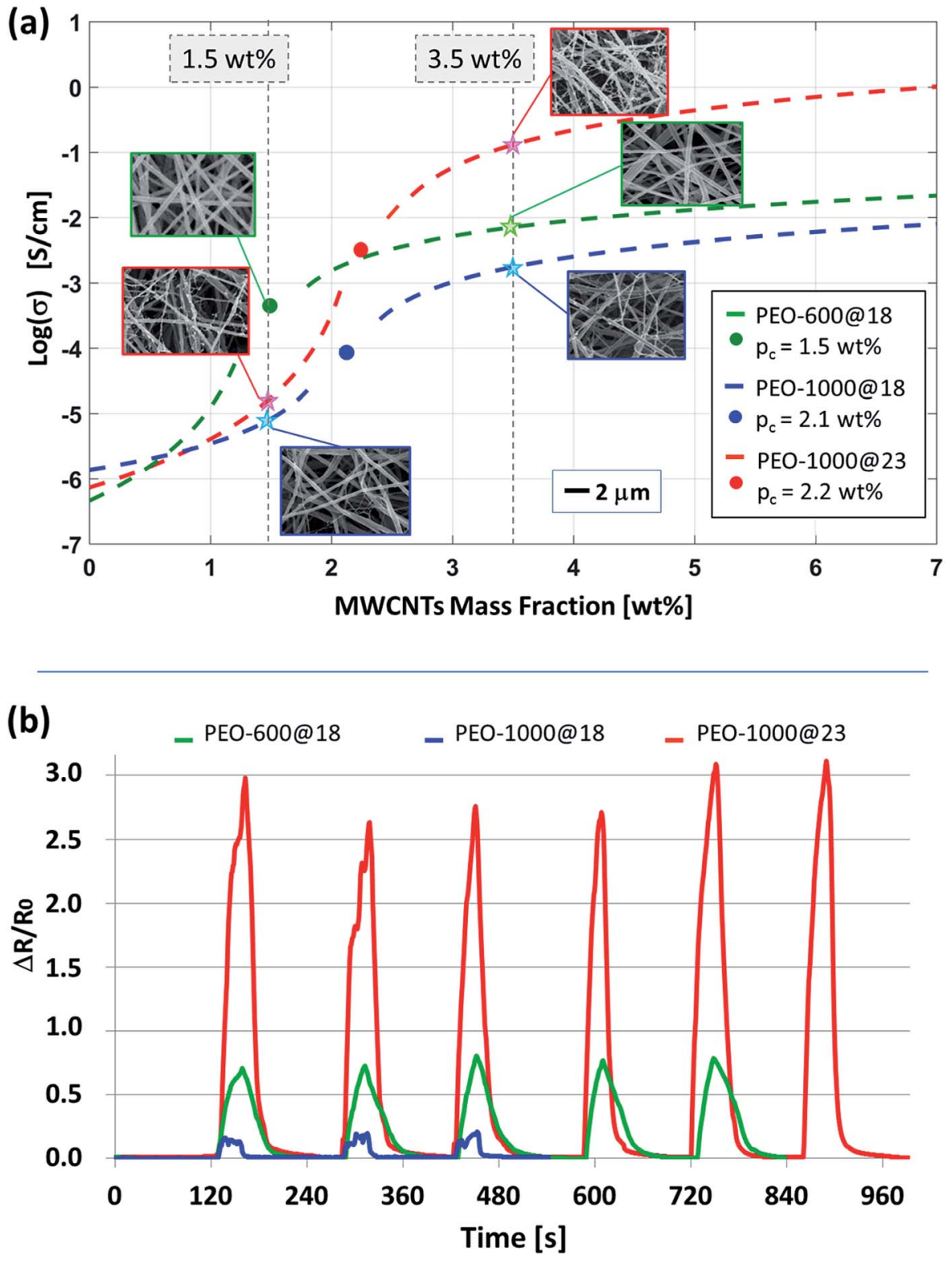

Fig. 4 (a) Experimental values of the electrical conductivity of composite nanofibers and nanofiber/nets for different concentration of MWCNTs. Three sets of samples are represented: green = PEO-600@18; blue = PEO-1000@18; red = PEO-1000@23 kV; the corresponding percolation thresholds are reported, and highlighted on the curves by coloured dots/stars. Pictures by FESEM are proposed as inserts, at two MWCNT concentrations, i.e. 1.5 and $3.5 \mathrm{wt} \%$, for the three sets of samples. (b) Relative differential resistance caused by humidity changes are reported versus time for the PEO-600@18, PEO-1000@18 and PEO-1000@23 samples.

thresholds, $p_{\mathrm{c}}$, are highlighted in same figure. The minimum $p_{\mathrm{c}}$ value of $1.5 \mathrm{wt} \%$ was found for samples PEO-600@18, while higher values of 2.2 and $2.1 \mathrm{wt} \%$, were estimated for samples PEO-1000@23 and PEO-1000@18, respectively.

It is interesting to notice that: (1) $p_{\mathrm{c}}$ increases for increasing values of the polymer average molecular weight; (2) for each MWCNT concentration, the conductivity of PEO-1000@18 is lower than the conductivity of the PEO-600@18 samples; (3) the PEO-1000@23 curve reaches the highest values of conductivity above percolation among the three set of samples. The latter two behaviours can be explained by comparing the morphology of the different samples.
In Fig. 4(a), two sets of FESEM images are proposed as inserts: they show the nanofibers and nanofiber/nets obtained for the three groups of samples, both at $1.5 \mathrm{wt} \%$ and at $3.5 \mathrm{wt} \%$ of MWCNTs. Comparing the images of nanofibers from samples PEO-600-1.5@18 and PEO-600-3.5@18 (with the green frames) to the pictures referring to the PEO-1000-1.5@18 and PEO-1000-3.5@18 nanofiber/nets (blue frames), it can be noticed that the latter samples show a nanofiber/net structure with a very low density (as described also in the ESM, Fig. S-3†), with less NFNs occupying pores than those visible in the PEO600@18 samples. In this case, the presence of low-density NFN is detrimental for the creation of percolation paths, since 
the distance among nanofibers is higher than for the other samples, so that higher concentration of MWCNTs are necessary to reach the percolation threshold. Moreover, above the percolation threshold, for each concentration of MWCNTs, the electrical conductivity remains lower than that of samples made with standard NF, thus demonstrating difficult electron transfer process among carbon nanotubes.

The PEO-1000@23 samples (red curve in Fig. 4(a)) exhibit a completely different electrical behaviour than the PEO1000@18 samples. Again we consider the morphology of the nanostructured materials to play a crucial role: indeed the former samples, processed at higher voltage show a more compact NFN structure, as shown in the pictures inserted in Fig. 4(a) with a red frame. In particular, by increasing the amount of MWCNTs in the material, pores are progressively occupied by NN and nanobeaded-NN. In addition, MWCNTs are present also in very small-size nanofibers, even in the secondary nanoweb, as shown in Fig. 2(d). This turns in the progressive improvement of the percolation networks while increasing the MWCNT content, thus increasing the probability of charge transfer across the mat of nanofibers, also thanks to the contribution of the nanonets. The result is that dense NFNs exhibit the onset of the electrical percolation at MWCNT concentration close to the value found for the less dense NFN, but the electrical conductivity above percolation is significantly improved. As a consequence, the final electrical behaviour of electrospinning-based composites can be tuned not only selecting its composition, by also by a careful design of the final nanostructure.

The designed material demonstrates new intriguing possibilities to optimize the NFN systems, in order to tune their morphology as well as their functional behaviour to match different potential application areas, as tissue engineering and environmental sensing. In both fields, the direct combined control of electrical conductivity and surface area of nanostructured mats can play a crucial role to gain optimal performance. ${ }^{22-25}$ With the aim to emphasize the potentiality of the NFN functional composite material, with unique morphological characteristics, properly combined with its tailored conduction properties, we performed preliminary tests to analyse its behaviour when exposed to humidity, investigating the effect of solvent-induced swelling on the conductivity.

The NFN functional composites designed in this work can be classified as conductive polymer composites, ${ }^{25,26}$ made of a polymeric matrix (i.e. the NFN) hosting an electricallyconductive filler. Exposure to humidity is expected to cause the polymeric nanofibers and NFN to swell by water adsorption, so increasing the distance among MWCNTs: if the concentration of MWCNTs is above the percolation threshold, the polymer swelling can directly affect conduction. Moreover, the worse is the quality of the percolation network, the lower will be the response of the material to the effect of swelling.

We performed the tests using the same sample architecture already used for the electrical characterizations of the material, directly exposing them to a vapour flow in air, generated by an aerosol system placed in proximity of the sample. We selected an aerosol system to avoid any effect of temperature on the samples during the tests. We worked at room temperature (i.e., $23{ }^{\circ} \mathrm{C}$ ) and the lowest value of the relative humidity was the ambient one (i.e., 23\% RH). A digital thermo-hygrometer (DTH) was placed in close proximity of the sample under investigation: $30 \mathrm{~s}$ were necessary to change the relative humidity from the lowest $(23 \% \mathrm{RH})$ to the highest $(60 \% \mathrm{RH})$ value, according to the DTH; after that time the humidity remained constant at $60 \%$ RH. Vapour was supplied to the sample in cycles: $30 \mathrm{~s}$ of exposure to humidity followed by 2 minutes of no exposure, working as a resting time necessary to restore the behaviour of the nanostructured samples to that shown before exposure to humidity.

The results are shown in Fig. 4(b), reporting the dimensionless relative differential resistance, $\Delta R / R_{0}$ as a function of time. $\Delta R / R_{0}$ is defined in eqn (3)

$$
\Delta R / R_{0}=\left(R-R_{0}\right) / R_{0}
$$

where $R$ is the actual resistance and $R_{0}$ is the baseline resistance of the composite materials prior to exposure to the humidity change. We analysed by this approach three sets of samples containing $3.5 \mathrm{wt} \%$ of MWCNTs: one made of NF only (i.e., PEO600-3.5@18), one with the low density NFN (i.e., PEO-10003.5@18) and the third with the high density NFN (i.e., PEO1000-3.5@23). The good reversibility in the response of all the samples is shown in Fig. 4(b).

The overall results are impressively in line with the expected behaviour of these systems. Indeed, samples PEO-1000-3.5@23 with a dense NFN structure, show a better behaviour than samples with NF only, because of the improved percolation pathways, giving higher electrical conductivity above percolation threshold. Dense NFN of PEO-1000-3.5@23 samples had a 4 times higher value of $\Delta R / R_{0}$ than samples PEO-600-3.5@18 with standard NF mats.

Analysing the curves in Fig. 4(b), the recovery speed $\left(R_{\mathrm{S}}\right)$ can be introduced, to help describing how fast the material regain its initial state after perturbation with humidity. $R_{\mathrm{S}}$ can be evaluated as the slope of the curves in the descendant part of each cycle. Analysing the materials by this approach, samples PEO-1000-3.5@18 and PEO-1000-3.5@23 show the fastest recovery process, with an average speed of $0.17 \mathrm{~s}^{-1}$ and $-0.14 \mathrm{~s}^{-1}$, respectively, while PEO-600 samples exhibit a quite slower recovery process, with an average $R_{\mathrm{s}}$ of $-0.05 \mathrm{~s}^{-1}$. This means that the NFNs, both the more and less dense one, help to improve the recovery process, and this can be explained considering that the nanowebs exhibit lower diameters than conventional NF mats. This turns in a faster humidity removal, that corresponds to a faster restoring of the percolation paths.

These results show that the electrical behaviour of composite NFNs can be directly tuned by the proper design of the nanonetting morphology, demonstrating a great potential of NFN composite materials.

\section{Conclusions}

In this work we have successfully demonstrated that a NFN functional composite material can be fabricated using water 
based solution of high molecular weight PEO and MWCNTs dispersed by Na-PSS, applying high voltages during the process. In particular, FESEM analyses highlighted the possibility to tune the morphology of the nanofiber-nets by selecting the working voltage applied during the ESN process: the higher the voltage the denser the resulting NFN. Moreover, two structures have been observed for the nanonetting, one made of very small size nanofibers and one with nanobeads, both due to a mechanism based on charge-unbalances during the ES process. TEM characterization evidenced the good orientation of MWCNTs in the NFN.

The electrical behaviour of the resulting PEO/MWCNT-NFN composite was analysed. The percolation theory was successfully applied, and a significant increase of the electrical conductivity was noticed, when a dense NFN was present in the material.

To investigate the direct effect of morphology on the final electrical behaviour of composite NFN, the response of the material to humidity-induced swelling was evaluated. Dense NFN significantly amplify the response of the material to external water vapour stimulus. The relative differential resistance of the denser NFN was four times higher than that shown by the standard composite NF. Moreover the recovery process was quite faster.

That experiment definitely demonstrated that the electrical behaviour of MWCNT based composite NFNs can be directly tuned by the proper design of the nanonets morphology.

Moreover, this tunable functional behaviour, when coupled to the biocompatibility and safety of PEO, results in a new amazing set of properties of the PEO/MWCNT-NFN, with great potential in fields as environmental sciences and tissue engineering.

\section{Conflicts of interest}

There are no conflicts of interest to declare.

\section{References}

1 B. Ding, C. Li, Y. Miyauchi, O. Kuwaki and F. Shiratori, Nanotechnology, 2006, 17, 3685.

2 X. Wang, B. Ding, G. Sun, M. Wang and J. Yu, Prog. Mater. Sci., 2013, 58, 1173.

3 S. Peng, G. Jin, L. Li, K. Li, M. Srinivasan, S. Ramakrishna and J. Chen, Chem. Soc. Rev., 2016, 45, 1225.
4 N. Bhardwaj and S. C. Kundu, Biotechnol. Adv., 2010, 28, 325.

5 C. Zhou, R. Chu, R. Wu and Q. Wu, Biomacromolecules, 2011, $12,2617$.

6 R. Nirmala, R. Navamathavan, S. J. Park and H. Y. Kim, NanoMicro Lett., 2014, 6, 89.

7 B. Ding, M. Wang, X. Wang, J. Yu and G. Sun, Mater. Today, 2010, 13, 16.

8 T. Amna, M. S. Hassan, F. A. Sheikh, H. K. Lee, K. S. Seo, D. Yoon and I. H. Hwang, Appl. Microbiol. Biotechnol., 2013, 97, 1725.

9 N. A. M. Barakat, M. A. Kanjwal, F. A. Sheikh and H. Y. Kim, Polymer, 2009, 50, 4389.

10 H. R. Pant, M. P. Bajgai, K. T. Nam, Y. A. Seo, D. R. Pandeya, S. T. Hong and H. Y. Kim, J. Hazard. Mater., 2011, 185, 124.

11 N. S. Sambudi, M. G. Kim and S. B. Park, Mater. Sci. Eng., C, 2016, 60, 518.

12 D. Kimmer, P. Slobodian, D. Petras, M. Zatloukal, R. Olejnik and P. Saha, J. Appl. Polym. Sci., 2009, 111, 2711.

13 D. Wu, T. Shi, T. Yang, Y. Sun, L. Zhai, W. Zhou, M. Zhang and J. Zhang, Eur. Polym. J., 2011, 47, 284.

14 X. Wang, Y. Si, X. Wang, J. Yang, B. Ding, L. Chen, Z. Hu and J. Yu, Nanoscale, 2013, 5, 886.

15 F. Wang, G. Wang, S. Yang and C. Li, Langmuir, 2008, 24, 5825-5831.

16 T. T. Tung, J. H. Yeon, T. Y. Kim and K. S. Suh, Synth. Met., 2010, 160, 1266-1272.

17 J. Xu, J. Li, Y. Wu and S. Bai, J. Mater. Sci.: Mater. Electron., 2014, 25, 3233-3241.

18 S. D. McCullen, D. R. Stevens, W. A. Roberts, S. S. Ojha, L. I. Clarke and R. E. Gorga, Macromolecules, 2007, 40, 997.

19 W. Salalha, Y. Dror, L. Khalfi, Y. Cohen, A. L. Yarin and E. Zussman, Langmuir, 2004, 20, 9852.

20 Y. Dror, W. Salalha, R. L. Khalfin, Y. Cohen, A. L. Yarin and E. Zussman, Langmuir, 2003, 19, 7012.

21 H. Fong, I. Chun and D. H. Reneker, Polymer, 1999, 40, 4585. 22 I. Fratoddi, A. Bearzotti, I. Venditti, C. Cametti and M. V. Russo, Sens. Actuators, B, 2016, 225, 96.

23 J. He, F. Xu, R. Dong, B. Guo and D. Li, Biofabrication, 2017, 9, 015007.

24 Y. Liu, X. Liang, S. Wang and K. Hu, J. Biomater. Tissue Eng., 2016, 6, 719.

25 E. Llobet, Sens. Actuators, B, 2013, 179, 32.

26 M. C. Lonergan, E. J. Severin, B. J. Doleman, S. A. Beaber, R. H. Grubbs and N. S. Lewis, Chem. Mater., 1996, 8, 2298. 